\title{
The effects of inescapable shock on appetitive motivation
}

\author{
MARK PLONSKY, DONALD A. WARREN, and ROBERT A. ROSELLINI \\ State University of New York, Albany, New York
}

\begin{abstract}
Exposure to inescapable shock is known to proactively interfere with the acquisition of instrumental responses to escape shock, as well as to obtain food. These effects have been termed learned helplessness. The present experiment investigated the possibility that the learned helplessness effect observed in an appetitive context may, at least in part, be due to the motivational effects of inescapable shock. The schedule-induced polydipsia paradigm, which is known to be sensitive to both deprivation level and incentive motivation, was used to assess the effects of inescapable shock on appetitive motivation. Despite the fact that learned helplessness was demonstrated in a shuttle escape task, no effect of inescapable shock was observed on polydipsia. Thus, the reinforcer generality of helplessness appears not to be due to shockinduced motivational factors.
\end{abstract}

Exposure to inescapable shock proactively interferes with the acquisition of instrumental responses to escape shock (for reviews, see Maier \& Jackson, 1979, and Maier \& Seligman, 1976). This effect has been termed learned helplessness and is postulated to result from an associative, as well as a motivational, deficit.

The learned helplessness effect also has been demonstrated when the reinforcer used in the test situation is appetitive rather than aversive. That is, interference with the acquisition of an instrumental response to obtain food has been demonstrated to result from exposure to inescapable shock (Caspy \& Lubow, 1981; Goodkin, 1976; Rosellini, 1978; Rosellini \& DeCola, 1981; Rosellini, DeCola, \& Shapiro, 1982).

In an effort to examine the effects of inescapable shock on appetitive motivation, Rosellini(1978) recorded the latency to ingest a food pellet in inescapably shocked animals and nonshocked controls. No significant difference was obtained between the groups. Similarly, Rapaport and Maier (1978) recorded both the latency to approach food and the amount consumed and also reported no differences between the groups. Thus, the interference observed in the appetitive studies noted above generally has been interpreted as resulting from an associative, rather than a motivational, effect of inescapable shock. Although such a conclusion is suggested by the findings of Rapaport and Maier (1978) and Rosellini (1978), the sensitivity of these procedures to detect differences in either motivational level or incentive value of the reinforcer may be limited.

The purpose of the present experiment was to employ a schedule-induced polydipsia (SIP) paradigm to assess more accurately any possible differences between

Reprint requests should be sent to R. A. Rosellini, Department of Psychology, State University of New York, Albany, New York 12222. inescapably shocked and nonshocked animals in appetitive motivation. The SIP paradigm (Falk, 1961) has been shown independently to be sensitive to an organism's deprivation level or hunger motivation (e.g., Freed \& Hymowitz, 1972; Roper \& Nieto, 1979), as well as to the incentive value or quality of the reinforcer (e.g., Rosellini \& Lashley, 1982).

\section{METHOD}

\section{Subjects}

Twenty-three male albino rats obtained from the Holtzman Company were used. All animals had previously served in a learned helplessness experiment (the details of which will be reported elsewhere) in which the animals were divided into two groups matched for weight and were given either inescapable shock (IS) or no shock (NS). Three weeks later, all animals were placed on a food-deprivation schedule and gradually were reduced to $80 \%$ of their free-feeding weights $($ mean $=514 \mathrm{~g}$ ) over a period of 1 week. The animals were individually housed with water continuously available, and the experiment was conducted during the later portion of the light phase of a 12-h light/dark cycle.

\section{Apparatus}

Six operant chambers, measuring $30.5 \times 25.5 \times 27.9 \mathrm{~cm}$, were used for polydipsia training. The front and back walls were constructed of aluminum, the side walls and ceiling of Plexiglas, and the floor of stainless steel rods $0.3 \mathrm{~cm}$ in diameter and spaced $1.3 \mathrm{~cm}$ apart. A food cup $(5.0 \times 2.5 \times 3.8 \mathrm{~cm})$ protruded from the center of the front wall $1.0 \mathrm{~cm}$ above the floor. A hole, $2.0 \mathrm{~cm}$ in diameter, was located $5.5 \mathrm{~cm}$ to the right of the food cup, and a $100-\mathrm{ml}$ graduated cylinder with a $2.5-\mathrm{mm}$ orifice was positioned $2.0 \mathrm{~mm}$ behind the aperture.

Four shock chambers, measuring $30.0 \times 20.5 \times 27.5 \mathrm{~cm}$, were used to administer inescapable shock. The front and back walls were constructed of aluminum, the side walls and ceiling of Plexiglas, and the floor of stainless steel rods $0.3 \mathrm{~cm}$ in diameter and spaced $1.3 \mathrm{~cm}$ apart. Also, a speaker was centered on the ceiling of each chamber.

Four shuttle boxes, measuring $45.7 \times 24.5 \times 21.6 \mathrm{~cm}$, were used for the learned helplessness shock escape test. The walls were constructed of aluminum, the ceiling of Plexiglas, and the floor of stainless steel rods $0.6 \mathrm{~cm}$ in diameter and spaced $1.9 \mathrm{~cm}$ 
apart. The center of the shuttle box contained an aluminum divider with an archway $7.0 \mathrm{~cm}$ high and $6.4 \mathrm{~cm}$ wide. A photocell was located $4.5 \mathrm{~cm}$ above the grid floor and $8.0 \mathrm{~cm}$ from each end wall.

Scrambled shock was delivered to the grid floors of the shock and shuttle boxes via solid-state shock sources (Coulbourn Instruments, Model E13-16). These boxes were located in the same room, which was different from that of the operant chambers. All of the experimental boxes were housed in sound- and lightattenuating chambers equipped with a $28-\mathrm{V}$ houselight and a ventilating fan. Control of apparatus and recording of data were accomplished through the use of a TRS- 80 microcomputer.

\section{Procedure}

On each of 2 consecutive days, the animals were placed in the operant chambers for $60 \mathrm{~min}$ and given access to $6045-\mathrm{mg}$ Noyes pellets massed in the food cup. The amount of water consumption during these sessions was recorded and served as the baseline intake level. The animals then received 15 daily $1 \mathrm{~h}$ polydipsia training sessions on a signaled fixed-time 60 -sec schedule. The signal consisted of a 100 -msec period of houselight absence that occurred simultaneously with pellet-dispenser operation. We followed the convention of Flory (1971) in defining SIP as a $200 \%$ increase in water intake from baseline level.

Immediately following Session 15 of polydipsia training, all animals were given an amount of food sufficient to maintain their $80 \%$ deprivation level. After consuming this food, the animals that had received shock several weeks earlier (the IS group, $N=11$ ) were given an additional 80 unsignaled inescapable shocks on a variable-time 60 -sec schedule (range $=5-115 \mathrm{sec}$ ). The shock intensity was $0.9 \mathrm{~mA}$, and the duration was $10 \mathrm{sec}$. The remaining animals (the NS group, $N=12$ ) were placed in the shock boxes for the same length of time as the IS group, but were not exposed to shock. White noise $(80 \mathrm{~dB})$ was present during this shock session. The following day, all animals received an additional session of polydipsia training (Session 16), during which an observer recorded the latency to pellet consumption for every third pellet for each animal (for a total of seven pellets per animal). The animals were again fed immediately after this session, and following consumption of the food, they were given a shuttle escape test to verify that the shock session did indeed produce helplessness in the IS group. This test consisted of 30 trials presented on a variable-time 60 -sec schedule (range $=5-115 \mathrm{sec}$ ). During the first 5 trials, the animal was required to shuttle from one side of the box to the other (fixed ratio 1, or FR 1) to terminate shock, whereas during the remaining trials, the animal was required to shuttle from one side to the other and back (FR 2). Each trial consisted of a maximum of $30 \mathrm{sec}$ of $0.6-\mathrm{mA}$ shock.

\section{RESULTS}

One animal in the NS group failed to develop SIP and was thus excluded from all analyses. Figure 1 presents the data from polydipsia training. As can be seen, mean levels of water consumption during the baseline sessions were low and did not differ between the groups $[t(20)=$ $1.27, \mathrm{p}=.215]$. Also, all animals increased their intake levels during the course of polydipsia training. More importantly, shock 1 month prior to polydipsia training had little effect on the development of SIP. Although there appears to have been a slight tendency for the NS animals to consume more water at asymptotic levels, an analysis of variance of the intakes on Sessions 1-15 as a function of shock condition revealed that neither the main effect of shock condition $[F(1,20)<1.0]$ nor its interaction with session $[F(14,280)=1.08, p=.377]$

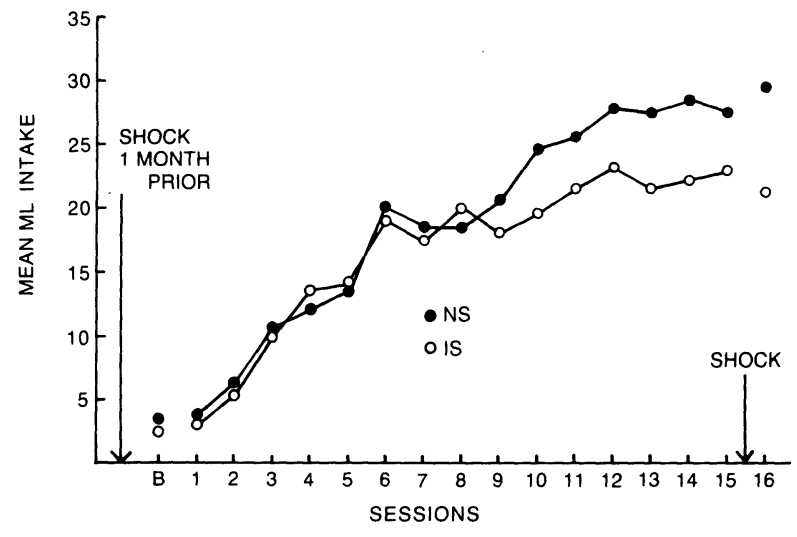

Figure 1. Mean milliliter intakes for the inescapable-shock (IS) and no-shock (NS) groups during the baseline (B) and polydipsia training sessions. The IS group received shock 1 month prior to training and again immediately following Session 15 of polydipsia training.

were statistically reliable. In addition, shock treatment following Session 15 did not appear to affect the maintenance levels of polydipsia. To assess this effect, the mean asymptotic performance (Sessions 12-15) was computed for each animal and analyzed in comparison with the Session 16 intakes. This analysis failed to reveal any statistically significant effects $[\mathrm{Fs}(1,20)<2.68$, ps $>.114]$. Analysis of the Session 16 latencies to eat also failed to reveal any significant effects [IS mean $=$ $0.67 \mathrm{sec}$, NS mean $=0.70, \mathrm{t}(20)<1.0]$.

Figure 2 presents the shuttle test performance as a function of shock condition and blocks of five trials. As can be seen, it appears that the IS animals demonstrated helplessness in comparison with the NS animals. Whereas the performance of the IS animals deteriorated over the course of the FR 2 trials, the NS animals remained consistent in their performance. As expected, the groups did not differ during the FR 1 trials $[t(20)<$

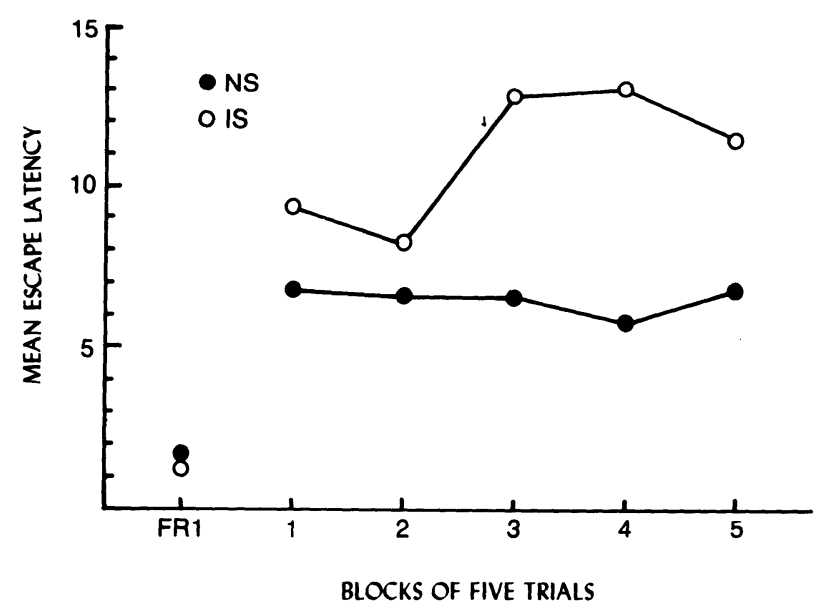

Figure 2. Mean escape latencies for the inescapable-shock (IS) and no-shock (NS) groups on the shuttle escape test in five trial blocks. 
1.0]. An analysis of variance of performance on the FR 2 trials revealed a significant shock condition $x$ trial block interaction $[F(1,80)=2.64, p=.039]$, as well as a marginally significant main effect of shock condition $[F(1,20)=3.14, p=.088]$.

\section{DISCUSSION}

The results of the present experiment indicate that inescapable shock given 1 month prior to SIP training does not significantly influence the development or maintenance of polydipsia. The results also demonstrate that inescapable shock given 1 day prior to testing produces learned helplessness as measured in the shuttle escape test, but does not influence maintenance levels of SIP. Since the SIP paradigm has been independently demonstrated to be sensitive to the organism's deprivational level (e.g., Freed \& Hymowitz, 1972; Roper \& Nieto, 1979), as well as to the incentive value of food (e.g., Rosellini \& Lashley, 1982), the present data strongly suggest that inescapable shock does not measurably influence appetitive motivation.

Rapaport and Maier (1978) and Rosellini (1978) have reported that prior exposure to shock does not affect measures such as the latency to consume or ingest food. Similarly, the present experiment was unable to find an effect of inescapable shock on appetitive motivation in a SIP paradigm. Thus, it appears unlikely that studies demonstrating interference with the acquisition of an appetitive response as a result of prior exposure to inescapable shock (Caspy \& Lubow, 1981; Goodkin, 1976; Rosellini, 1978; Rosellini \& DeCola, 1981; Rosellini et al., 1982) can be interpreted in terms of alterations in appetitive motivation.

Furthermore, the findings of Rosellini et al. (1982) indicate that the deficits observed in an appetitive context cannot be explained simply on the basis of inescapable shock's producing a decrease in the motivation to initiate responding. They observed deficits in the acquisition of an appetitive response choice discrimination task as a result of exposure to inescapable shock. Since analysis of performance on this task includes only trials in which the animal makes a response, the observed deficit cannot be explained in terms of inescapable shock's producing a decrease in the motivation to initiate responding. Therefore, the learned helplessness effect observed in an appetitive context is most probably due to an associative deficit. It must be acknowledged, however, that these effects may also be due to alterations in attention resulting from inescapable shock. Additional research is needed to clarify this issue further.

\section{REFERENCES}

CASPY, T., \& LuBow, R. E. (1981). Generality of US preexposure effects: Transfer from food to shock or shock to food with and without the same response requirement. Animal Learning \& Behavior, 9, 524-532.

FALK, J. L. (1961). Production of polydipsia in normal rats by an intermittent food schedule. Science, 133, 195-196.

FLORY, R. K. (1971). The control of schedule-induced polydipsia: Frequency and magnitude of reinforcement. Learning and Motivation, 2, 215-227.

Freed, E., \& Hymowitz, N. (1972). Effects of schedule, percent body weight, and magnitude of reinforcer on acquisition of schedule-induced polydipsia. Psychological Reports, 31, 95-101.

GoodKIn, F. (1976). Rats learn the relationship between responding and environmental events: An expansion of the learned helplessness hypothesis. Learning and Motivation, 7, 382-393.

MAIER, S. F., \& JACKSON, R. L. (1979). Learned helplessness: All of us were right (and wrong): Inescapable shock has multiple effects. In G. H. Bower (Ed.), The psychology of learning and motivation (pp. 155-218). New York: Academic Press.

Maier, S. F., \& Seligman, M. E. P. (1976). Learned helplessness: Theory and evidence. Journal of Experimental Psychology: General, 105, 3-46.

RAPAPORT, P. M. \& MAIER, S. F. (1978). Inescapable shock and food-competition dominance in rats. Animal Learning \& Behavior, 6, 160-165.

ROPER, T. J., \& NiEto, J. (1979). Schedule-induced drinking and other behavior in the rat, as a function of body weight deficit. Physiology \& Behavior, 23, 673-678.

Rosellini, R. A. (1978). Inescapable shock interferes with the acquisition of a free appetitive operant. Animal Learning \& Behavior, 6, 155-159.

Rosellini, R. A., \& DeCola, J. P. (1981). Inescapable shock interferes with the acquisition of a low-activity response in an appetitive context. Animal Learning \& Behavior, 9, 487-490.

Rosellini, R. A., DeCola, J. P., \& Shapiro, N. R. (1982). Cross-motivational effects of inescapable shock are associative in nature. Journal of Experimental Psychology: Animal Behavior Processes, 8, 376-388.

Rosellini, R. A., \& LAshley, R. L. (1982). The opponentprocess theory of motivation: VIII. Quantitative and qualitative manipulations of food both modulate adjunctive behavior. Learning and Motivation, 13, 222-239.

(Manuscript received for publication December 16, 1983.) 